\title{
Deciphering the therapeutic mechanisms of Xiao- Ke-An in treatment of type 2 diabetes in mice by a Fangjiomics approach
}

\author{
Zhen-zhong YANG ${ }^{1}$, Wei $\mathrm{LIU}^{1}$, Feng ZHANG ${ }^{1}$, Zheng $\mathrm{LI}^{2, *}$, Yi-yu CHENG ${ }^{1}$ \\ ${ }^{1}$ Pharmaceutical Informatics Institute, College of Pharmaceutical Sciences, Zhejiang University, Hangzhou 310058, China; ${ }^{2}$ State Key \\ Laboratory of Modern Chinese Medicine, Tianjin University of Traditional Chinese Medicine, Tianjin 300193, China
}

\begin{abstract}
Aim: Xiao-Ke-An (XKA) is a traditional Chinese medicine (TCM) formula for the treatment of type 2 diabetes (T2D), and the effective ingredients and their targets as well as the mechanisms of XKA remain to be elucidated. In this study we investigated the therapeutic mechanisms of XKA in the treatment of T2D in mice using a Fangjiomics approach.

Methods: KKAy mice feeding on a high-fat diet were used as models of T2D, and were orally treated with XKA $\left(0.75\right.$ or 1.5 g.kg-1 $\left.\cdot \mathrm{d}^{-1}\right)$ for $32 \mathrm{~d}$. Microarray mRNA expression data were obtained from the livers of the mice. Differentially expressed genes (DEGs) were identified by reverse rate analysis and ANOVA analysis. The compounds in XKA were identified by LC-MS analysis or collected from TCM databases. The relationships between the compounds and targets were established by combining the DEGs with information derived from mining literature or herb target databases. Relevant pathways were identified through a Kyoto Encyclopedia of Genes and Genomes pathway enrichment analysis using WebGestalt.

Results: The compound-target-pathway network based on compounds identified by LC-MS analysis (NCA) included 20 constituent compounds, 46 targets and 36 T2D-related pathways, whereas the compound-target-pathway network based on compounds collected from databases (NCD) consisted of 40 compounds, 68 targets and 21 pathways. In the treatment of T2D, XKA might act mainly by improving carbohydrate and lipid metabolism, as well as ameliorating insulin resistance, inflammation and diabetic vascular complications.

Conclusion: The network-based approach reveals complex therapeutic mechanisms of XKA in the treatment of T2D in mice that involve numerous compounds, targets, and signaling pathways.
\end{abstract}

Keywords: type 2 diabetes; Xiao-Ke-An; traditional Chinese medicine; network pharmacology; Fangjiomics

Acta Pharmacologica Sinica (2015) 36: 699-707; doi: 10.1038/aps.2014.138; published online 11 May 2015

\section{Introduction}

Type 2 diabetes (T2D) is one of the most rapidly increasing diseases throughout the world as a result of high rates of obesity, increased urbanization and lifestyle changes ${ }^{[1]}$. T2D is a chronic, complex and progressive disease involving multiple pathophysiological defects, such as insulin resistance and $\beta$-cell dysfunction ${ }^{[2,3]}$. Exercise, diet management and the administration of oral anti-diabetic agents are widely applied to control glucose homoeostasis in both the postprandial and fasting states ${ }^{[4]}$. Many oral medications for T2D are currently available, the majority of which are single-target drugs. However, the prolonged administration of these medicines could lead to numerous adverse effects, long-term toxicity, or drug

\footnotetext{
* To whom correspondence should be addressed.

E-mail lizheng1@gmail.com

Received 2014-08-28 Accepted 2014-10-30
}

tolerance ${ }^{[5]}$. For example, thiazolidinediones (TZDs), which are peroxisome proliferator-activated receptor- $\gamma$ (PPAR- $\gamma$ ) agonists, could cause weight gain and fluid retention. Moreover, rosiglitazone therapy has been associated with a significantly increased risk of cardiovascular events ${ }^{[6]}$. Metformin may cause gastrointestinal disturbances ${ }^{[7]}$ and lactic acidosis ${ }^{[8]}$. Multi-target drugs and formulas, eg, traditional Chinese medicine (TCM), show distinctive superiority in the treatment of complex diseases compared to single-target drugs ${ }^{[9,10]}$. Fangji (a TCM formula) consists of multiple ingredients or modalities and is commonly used in clinical practice to treat different diseases in China. For example, Fangji has been used for thousands of years to treat T2D, which belongs to the scope of 'Xiaoke' in TCM. Abundant clinical practical experience has been accumulated, and some Fangjis exhibit sound clinical efficacy in the treatment of this disease. However, its detailed therapeutic mechanisms remain unclear. 
Fangjiomics is the science of designing effective mixtures of Fangjis and elucidating their modes of action often by combining omics technologies ${ }^{[11]}$, and network pharmacology offers a network perspective to understand the relationships between drugs and targets. By integrating system-level omics data (eg, transcriptome expression data) with network-based analyses, a new avenue has emerged to help explain the complex therapeutic mechanisms of Fangji.

Xiao-Ke-An (XKA) is a Chinese medicine for T2D approved by China Food and Drug Administration. However, the effective ingredients and their targets as well as the therapeutic mechanisms of XKA remain to be systematically elucidated. In this study, the therapeutic mechanisms of XKA were investigated using a Fangjiomics approach, as shown in Figure 1. By combining compounds of XKA with their associated targets and pathways, compound-target-pathway networks were constructed to illustrate the molecular mechanisms of XKA for T2D from a network perspective. This study provides a novel approach for studying the mechanisms of Fangji in the treatment of complex diseases.

\section{Materials and methods Materials}

XKA samples were obtained from Jilin Tonghua Huaxia Pharmaceutical Co Ltd (Jilin, China). Eight-week-old male KKAy mice were obtained from Beijing HFK Bioscience Co, Ltd (Beijing, China). Age-matched male non-diabetic C57BL/6J mice were obtained from Shanghai SLAC Laboratory Animal Co, Ltd (Shanghai, China).

\section{Animal experiment}

All mice were maintained in an air-conditioned room (temperature, $22 \pm 2{ }^{\circ} \mathrm{C}$; relative humidity, $55 \% \pm 15 \%$ ) with a $12-\mathrm{h}$ light/dark cycle and were allowed ad libitum access to food and water. The KKAy mice were used as models of T2D and were fed a high-fat diet. The C57BL/6J mice were fed normal diets and were used as non-diabetic controls. The Animal Ethics Review Committees of Zhejiang University approved all the procedures.

After 2 weeks of acclimation, KKAy mice were assigned to 4 groups according to their fasting blood glucose values. The KKAy mice received the once-daily oral administration of either distilled water (model group), XKA (1.5 g/ kg), XKA $(0.75 \mathrm{~g} / \mathrm{kg})$, or metformin $(250 \mathrm{mg} / \mathrm{kg})$. The C57BL/6J mice were gavaged with distilled water as a normal group.

After $32 \mathrm{~d}$ of drug administration, all the mice were sacrificed in the fed state, and the livers were collected and stored in liquid nitrogen. The metformin treatment group was used as the positive control. The therapeutic effects of XKA (both $1.5 \mathrm{~g} / \mathrm{kg}$ and $0.75 \mathrm{~g} / \mathrm{kg}$ ) and metformin on controlling T2D were tested, and the results will be reported in a separate paper. XKA $(1.5 \mathrm{~g} / \mathrm{kg})$ treatment exhibited more potent antidiabetic effects than XKA $(0.75 \mathrm{~g} / \mathrm{kg})$. Therefore, the livers from the normal, model and XKA $(1.5 \mathrm{~g} / \mathrm{kg})$ treatment groups were used for further microarray experiments in this study.

\section{Microarray experiment}

Total RNA was extracted using the mirVana ${ }^{\mathrm{TM}}$ PARIS $^{\mathrm{TM}}$ Kit (Ambion, Austin, TX, USA) following the manufacturer's instructions. RNA quality was evaluated using an Agilent 2100 Bioanalyzer (Agilent Technologies, Santa Clara, CA, USA). Only RNAs with RNA integrity numbers (RINs) $\geq 7.0$ and $28 \mathrm{~S} / 18 \mathrm{~S}$ ratios $\geq 0.7$ were used for the microarray experiments.

A whole-genome microarray analysis was performed using the Affymetrix mouse 4302.0 array. The processes of sample labeling, microarray hybridization and washing were performed according to the manufacturer's standard protocols. Total RNA was transcribed to double strand cDNA, and then the biotin-labeled cRNA was synthesized. The labeled

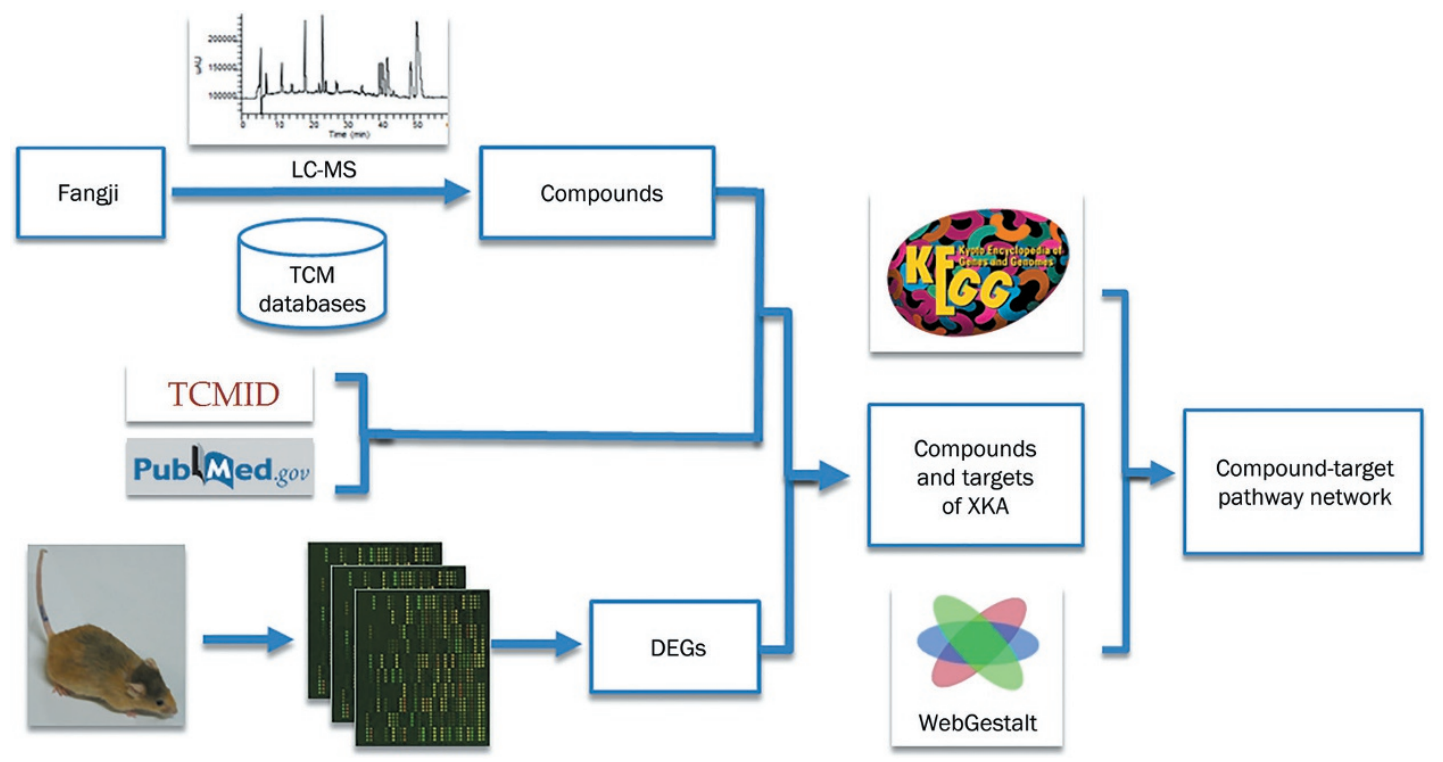

Figure 1. The framework of the Fangjiomics study of XKA. 
cRNA was hybridized onto the microarray. The washing and the staining were performed following the manufacturer's instructions. Slides were scanned by a GeneChip Scanner 3000 (Affymetrix, Santa Clara, CA, USA) and Command Console Software 4.0 with the default settings. Five mice per group were used for the microarray experiments. The dataset was deposited as CEL files to GEO under access number GSE62087.

\section{Microarray data analysis}

The raw data were analyzed using ArrayTrack 3.5.0 $0^{[12]}$, a Javabased microarray analysis tool developed by the US FDA. Global scaling normalization was performed with Median Scaling Normalization in ArrayTrack 3.5.0 using a target median value of 1000 . The reversal effect of XKA on gene expression in the diabetic models was evaluated with a reverse rate (RR) analysis ${ }^{[13]}$. This effect was calculated via the following equation: $R R=\left(M_{i}-D_{i}\right) /\left(M_{i}-N_{i}\right)$, in which $N_{i}, M_{i}$, and $D_{i}$ are the average expression levels of gene $i$ in the normal group, model group, and XKA treatment group, respectively. Differentially expressed genes (DEGs) were identified using $R R>0.5$ and an analysis of variance (ANOVA) among each group with a cutoff $P$ value $\leq 0.05$. The gene expression levels of these DEGs in T2D models were reversed due to the effects of XKA.

\section{Network construction}

Two different approaches were adopted to construct the compound-target-pathway network. In the first method, the compounds in XKA identified by liquid chromatography-mass spectrometry (LC-MS) analysis (the process will be reported in a separate paper) were introduced. These compounds were then used separately to identify their associated targets. The targets of the compounds identified by the LC-MS analysis were searched by manually mining the existing literature in PubMed. If a gene or protein was reported to be affected by a compound in XKA in the abstract of an article, it was considered a potential target of the compound. The DEGs confirmed by mining the literature were assessed as potential targets of XKA.

Relevant pathways were further identified through a Kyoto Encyclopedia of Genes and Genomes (KEGG) ${ }^{[14]}$ pathway enrichment analysis of the potential targets of XKA using WebGestalt ${ }^{[15]}$. The pathways closely related to diabetes were selected for further analysis.

In the second method of network construction, TCM and herb databases, including TCM-ID ${ }^{[16]}$, HIT ${ }^{[17]}$, and TCM@Taiwan $^{[18]}$, were used to identify the chemicals in XKA by searching the constituent herbs of XKA (Rehmanniae radix, Anemarrhenae rhizoma, Coptidis rhizoma, Lycii cortex, Lycii fructus, Polygonati odorati rhizoma, Ginseng radix et rhizoma and Salviae miltiorrhizae radix et rhizoma). The Traditional Chinese Medicine Integrated Database (TCMID) ${ }^{[19]}$ records TCM-related information collected from different resources and through text-mining methods. This database was used in this study to investigate the targets of the compounds. The DEGs confirmed by the herb target database were assessed as potential targets of XKA. Relevant pathways were identified as described above.
Based on these results, the compound-target-pathway network with compounds identified by LC-MS analysis (NCA) and the compound-target-pathway network with compounds collected from databases (NCD) were separately constructed using Cytoscape version 2.8.2 ${ }^{[20]}$. If the interaction between a compound and a target was described in the literature or herb target database, the component and the associated target were connected with a line. The edge between the target and pathway was constructed with the relationship obtained in the pathway enrichment analysis.

\section{Results}

We first applied a principal component analysis (PCA) of the data to the global gene expression data. The PCA view using the principal component 1 (PC1) and principal component 5 (PC5) for the global gene expression data from the samples of normal, model and XKA-treated groups is shown in Figure 2. In the plot, all of the normal samples are clustered together on the right, whereas the T2D model ones are scattered on the left. The XKA-treated ones are located between the normal and the model samples. This result suggested that the XKAtreated samples exhibited a certain degree of reversion from the disease state to the normal state at the global gene expression level along the PC1 direction.

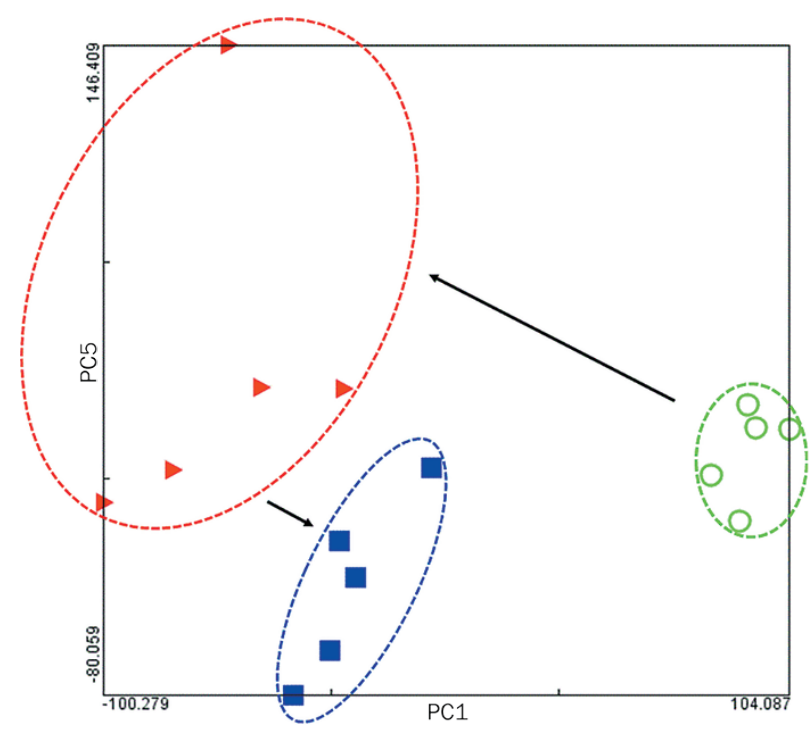

Figure 2. Principal component analysis (PCA) of the data from the global gene expression profile. Green circles represent the normal samples; red triangles represent the model samples; and blue squares represent the XKA-treated samples.

According to the Orthologene database in ArrayTrack, the mouse gene names were converted to the human names. In total, 1743 genes (2575 probe sets) were found to be differentially expressed, with RR $>0.5$ and $P$ value $\leq 0.05$ after treatment with XKA. A hierarchical cluster analysis (HCA) was performed on the DEGs, as shown in Figure 3. All the replicates within each experimental group clustered together, indicating 

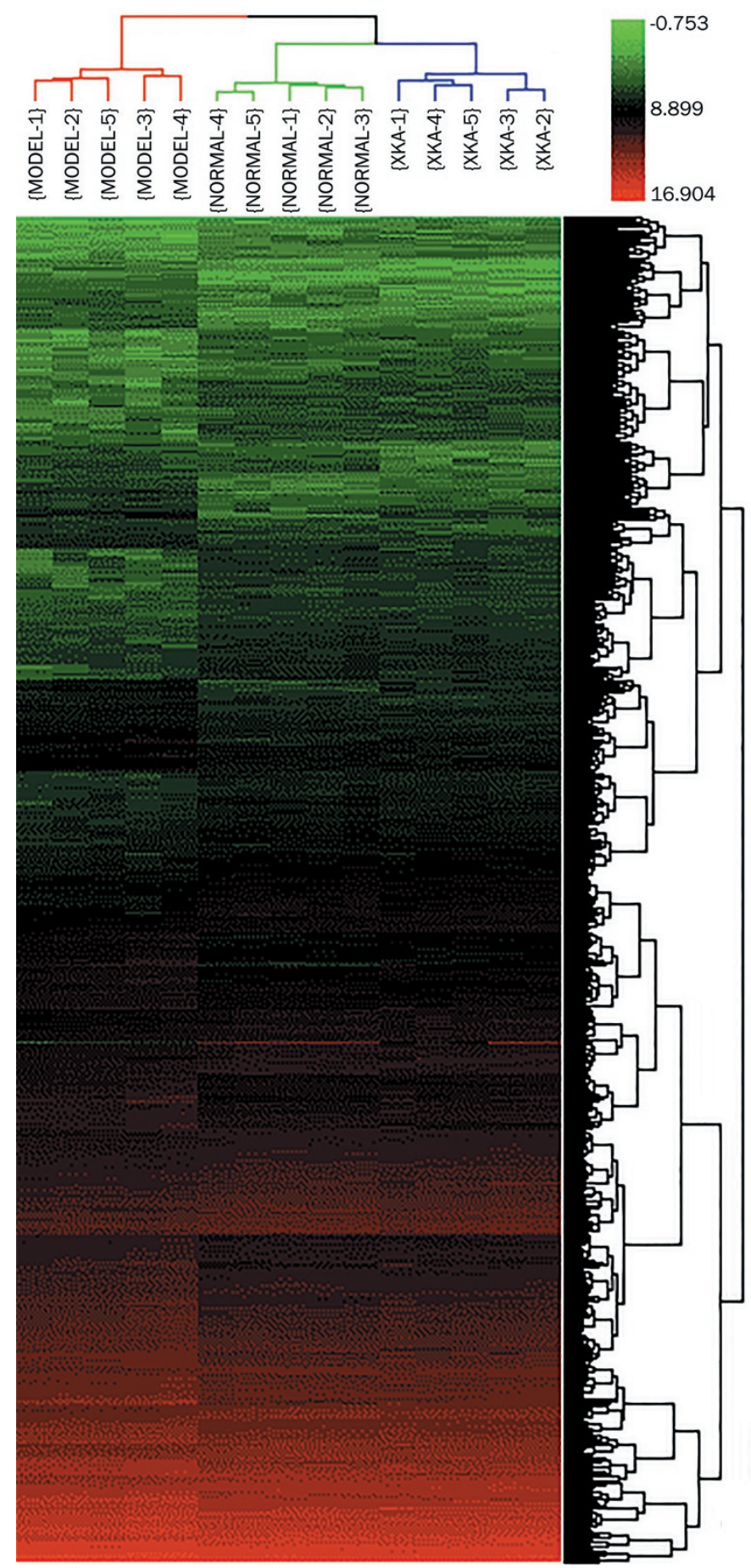

Figure 3. Heatmap and dendrogram of differentially expressed genes. The heatmap shows the expression levels of the 2575 probe sets. Red represents high expression and green represents low expression.

the high quality of the microarray data.

In these DEGs, 46 genes were found to be potential targets of the 20 constituent compounds of XKA identified by the LC-MS analysis and confirmed by mining the literature. Moreover, 36 T2D-associated pathways were functionally enriched by these 46 genes. Detailed information about the literature is presented in Table S1.

The top 10 compounds with the most targets are listed in Table 1. Berberine had 21 targets, and the target numbers of ginsenoside Rg1, ginsenoside Rb1, ginsenoside Re and ginsenoside $\mathrm{Rd}$ were $17,14,10$, and 8 , respectively.

Based on the whole-genome expression data and the target information for the LC-MS-identified constituent compounds in XKA, a compound-target-pathway network (NCA) was constructed. NCA consisted of 102 nodes and 290 edges, as illustrated in Figure 4A and Table 2.

Among the XKA constituent compounds identified by mining databases, some widely distributed compounds, such as palmitic acid, were excluded. A total of 40 constituent compounds were gathered from TCM-ID, HIT and TCM@Taiwan for further use, and 68 DEGs were identified as potential targets of the 40 constituent compounds by mining the drug target database. A total of $21 \mathrm{~T} 2 \mathrm{D}$-associated pathways were enriched, as shown in Table 2.

Based on the results of the microarray gene expression analysis and the target information of XKA's databases derived constituent compounds from the drug target database, the compound-target-pathway network with compounds collected from databases (NCD) was also built. NCD consisted of 129 nodes and 204 edges, as illustrated in Figure 4B.

Many potential targets were regulated by more than one compound in both NCA and NCD. For instance, CASP3 was influenced by 14 compounds in NCA and 15 in NCD. For some compounds, the number of potential targets differed between these two networks. superoxide dismutase 1 (SOD1) was influenced by 13 compounds in NCA, whereas the number was only 6 in NCD. However, SOD1 was targeted by catalpol, danshensu and mangiferin in both NCA and NCD.

Berberine derived from Coptidis rhizoma, ginsenosides from Ginseng radix et rhizome, and polyphenolic substrates from Salviae miltiorrhizae radix et rhizoma influenced most of the targets of XKA in NCA. In NCD, berberine, betaine derived from Lycii cortex, ginsenosides, tanshinones and polyphenolic substrates derived from Salviae miltiorrhizae radix et rhizoma were the most influential compounds. Common targets regulated by the same compound of XKA were summarized from both networks (NCA and NCD) and are listed in Table S2.

Pathways related to energy metabolism, insulin resistance, oxidative stress, inflammation and apoptosis processes were included in both NCA and NCD. Therefore, the complex therapeutic mechanisms of XKA for T2D might mainly involve improving energy metabolism and ameliorating insulin resistance, oxidative stress and inflammation. Pathways associated with vascular disease, such as the VEGF and ErbB signaling pathways, were also enriched in both networks. This result suggests a potential use of XKA to attenuate diabetic complications, eg, diabetic retinopathy and diabetic nephropathy.

\section{Discussion}

Two compound-target-pathway networks, ie, NCA and NCD, were constructed separately in this study. Because of the different origins of the constituent compounds in XKA, these two 
Table 1. The top 10 compounds with the most targets.

\begin{tabular}{lcl}
\hline \multicolumn{1}{c}{ Compound } & Number of targets & Target \\
\hline Berberine & 21 & $\begin{array}{l}\text { INSR SLC2A1 FOX03 NANOG HIF1A VEGFA CASP3 TP53 MAPK8 SOD1 SOD2 BAX TFRC GPT2 GOT2 XIAP } \\
\text { VCAM1 SRC CCNB1 WEE1 PPARGC1A }\end{array}$ \\
Ginsenoside Rg1 & 17 & RHOB IQGAP1 LAMA4 TP53 CREB1 BAX CASP3 HIF1A PIK3CA VEGFA CTNNB1 IGF1 MAPK8 TH IL4 SOD1 SOD2 \\
Salvianolic acid B & 15 & SOD1 SOD2 BAX CASP3 TP53 ROCK2 MAP2K1 MAP2K2 ATP1B1 ATP1A1 MAPK8 NFE2L2 VCAM1 EIF2A VEGFA \\
Ginsenoside Rb1 & 14 & CASP3 MAPK8 BAX IRAK1 VEGFA HIF1A VCAM1 SOD1 SOD2 PIK3CA NFE2L2 TP53 IL4 CREB1 \\
Catalpol & 14 & IL4 MAPK8 SOD1 SOD2 ATP2A1 ATP1B1 ATP1A1 BAX CAMK2B AGER CREB1 VEGFA TH CASP3 \\
Ginsenoside Re & 10 & CASP3 BAX PIK3CA MAPK8 IRAK1 IL4 FABP4 SOD1 SOD2 TH \\
Danshensu & 8 & NFE2L2 VEGFA CASP3 BAX AGER SOD1 SOD2 MAPK8 \\
Salvianolic acid A & 8 & CCNB1 TP53 CASP3 NFE2L2 VEGFA VCAM1 PPARGC1A BAX \\
Ginsenoside Rd & 8 & SOD1 SOD2 MAPK8 CASP3 TRPM7 BAX CREB1 IL4 \\
Mangiferin & 8 & IL4 NFE2L2 SOD1 SOD2 BAX AGER MAPK8 LPL \\
\hline
\end{tabular}

Table 2. Comparison between NCA and NCD.

\begin{tabular}{lrr}
\hline & NCA & NCD \\
\hline Total nodes & 102 & 129 \\
Compounds & 20 & 40 \\
Targets & 46 & 68 \\
Pathways & 36 & 21 \\
Total edges & 290 & 204 \\
Edges linking compounds and targets & 146 & 136 \\
Edges linking targets and pathways & 144 & 68 \\
\hline
\end{tabular}

networks demonstrated different coverage of the therapeutic mechanisms of XKA. The constituent compounds of NCA derived from the LC-MS analysis were relatively more reliable. The major components of XKA were included, with the majority of therapeutic mechanisms represented. However, many trace ingredients might not be detected with this method. The TCM databases collect numerous data from scientific publications, and the information about the compounds of the constituent herbs in XKA was much more comprehensive. Thus, NCD would reveal more comprehensive and integrated therapeutic mechanisms of XKA.

In both NCA and NCD, berberine was one of the most intriguing compounds, regulating 21 (in NCA) and 25 (in NCD) DEGs. Berberine is a star molecule originally derived from the Chinese herb Coptis Chinensis and is usually used as an antibiotic agent for treating gastrointestinal infections. Since the accidental discovery of the hypoglycemic effect of berberine, numerous studies in animal and cell line models, as well as clinical investigations, have been reported. Some other Fangjis containing berberine have also been reported to exhibit anti-diabetic effects ${ }^{[21,22]}$. Berberine may improve insulin sensitivity through the induction of insulin receptor expression $^{[23,24]}$. In this study, XKA reversed the decreased mRNA expression of the insulin receptor (INSR), which might be attributed to the effect of berberine. Additionally, a portion of the hypoglycemic effects of berberine might be attributed to its improvement of glucose transport ${ }^{[25,26]}$. XKA reversed the decreased mRNA expression of solute carrier family 2 member 1 (SLC2A1), which might contribute to this effect of XKA.

Betaine is a trimethyl derivative of glycine originally derived from Lycii cortex. Dietary betaine supplementation significantly decreases the mRNA levels of lipoprotein lipase $(\mathrm{LPL})^{[27]}$. Therefore, betaine might contribute to the improvement in lipid metabolism by XKA.

Mangiferin, a xanthonoid from Anemarrhenae rhizoma, significantly reduced the total inflammatory cell counts and down-regulated the levels of one group of cytokines, including Th2-related IL-4, in mouse model of allergic asthma ${ }^{[28]}$. In lipopolysaccharide-stimulated alveolar macrophages, catalpol inhibited TNF- $\alpha$, IL-6, IL-4 and IL-1 $\beta$ production ${ }^{[29]}$. Therefore, these compounds might contribute to the anti-inflammatory effects of XKA.

Oxidative stress plays a prominent role in glucose metabolism abnormalities, which is one of the risk factors associated with insulin resistance, T2D and diabetes complications. SODs, the primary cellular defense against superoxide radicals, catalyze the conversion of superoxide to hydrogen peroxide, which can be subsequently converted to water by other antioxidants $^{[30]}$. Patients with insulin resistance had decreased plasma SOD activity ${ }^{[31]}$. The genetic ablation of SOD1 led to glucose intolerance, which was associated with $\beta$-cell insulin secretion reduction and $\beta$-cell volume decrease ${ }^{[32]}$. Catalpol has been reported to exert significant cytoprotective effects on astrocytes by suppressing the production of free radicals and elevating antioxidant capacity. This process was partly dependent on the elevated activities of antioxidases, including $\mathrm{SOD}^{[33]}$. In a rat model of hypertrophy, danshensu prevented cardiac ligation/reperfusion injury and improved cardiac function, which partially resulted from the increased serum activity of SOD by the antioxidants ${ }^{[34]}$. The oral administration of mangiferin to diabetic rats significantly restored the activity of antioxidant enzymes, such as SOD ${ }^{[35]}$. Ginsenoside Rd exhibited anti-inflammatory activities by elevating the activity of antioxidant enzymes, including SOD, in vivo ${ }^{[36]}$. Nuclear factor erythroid 2-related factor-2 (NRF2, also known 


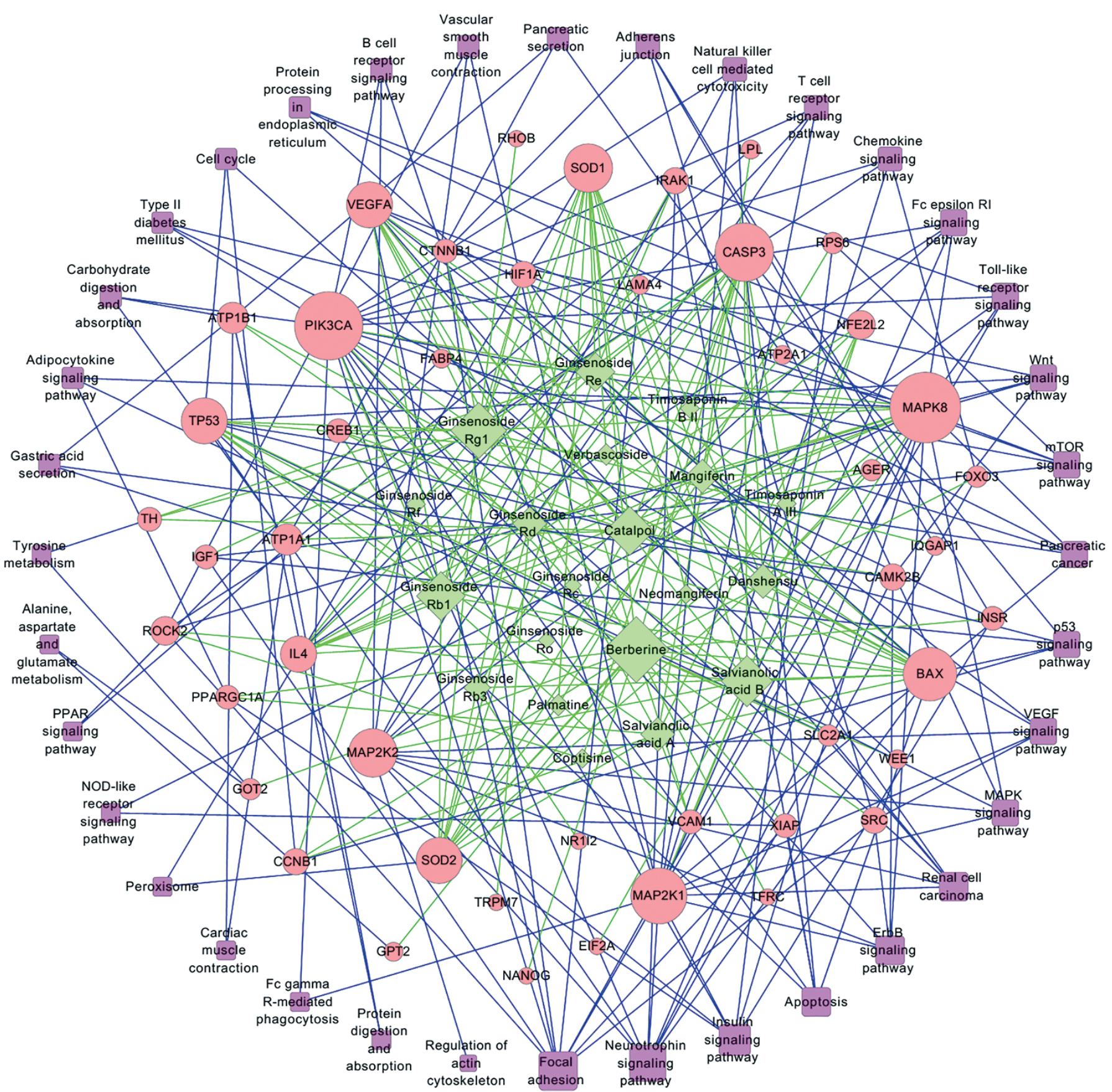

Figure 4A. The compound-target-pathway networks of XKA in the treatment of T2D. (A) The compound-target-pathway network with compounds identified by LC-MS analysis (NCA).

as NFE2L2) is a transcription factor that regulates the expression and coordinated induction of numerous reactive oxygen species-detoxifying and antioxidant genes ${ }^{[37]}$. The up-regulation of NRF2 alleviates oxidative stress and reduces the risk of chronic diabetic complications ${ }^{[38]}$. Danshensu pretreatment significantly attenuated 6-hydroxydopamine-induced reactive oxygen species (ROS) production in PC12 cells, which was mediated by activating the nuclear translocation of $\mathrm{NRF}^{[39]}$. Salvianolic acid A increased the protein expression of NRF2 to inhibit $\mathrm{H}_{2} \mathrm{O}_{2}$-mediated damage in primary cultured retinal pigment epithelial cells ${ }^{[40]}$. Salvianolic acid B attenuated toxininduced neuronal damage by increasing the expression and nuclear translocation of NRF2 ${ }^{[41]}$. Therefore, these compounds may be the major components of XKA that improve the activities of antioxidant enzymes, reduce oxidative stress and prevent metabolic dysfunction.

Atherosclerosis is one of the main causes of death in patients with diabetes. Circulating leukocytes adhering to the vascular endothelium at the atherosclerosis sites plays a critical role in the development of atherosclerosis. Adhesion molecules, such 


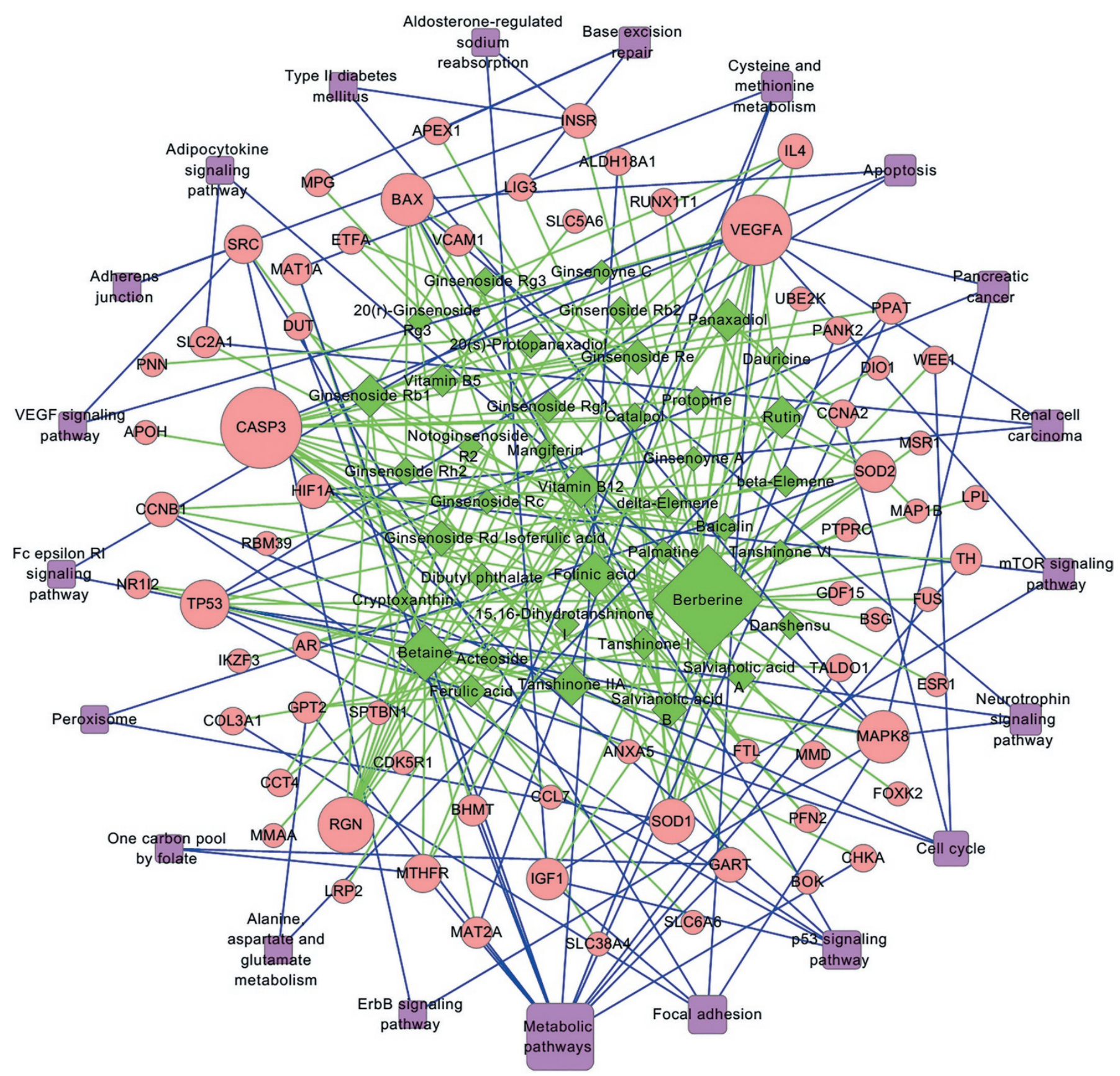

Figure 4B. (B) The compound-target-pathway network with compounds collected from databases (NCD). Green diamond nodes represent compounds; pink circle nodes represent target genes; purple rounded square nodes represent T2D-associated pathways. The node size is proportional to the degree of the node. Green edges link compounds to target genes and blue edges link target genes to T2D-associated pathways.

as intercellular adhesion molecule-1 (ICAM1) and vascular cell adhesion molecule-1 (VCAM1), play an important role in the process of leukocyte recruitment. The serum VCAM1 concentration of diabetic patients with macroangiopathy was elevated $^{[42]}$. Serum VCAM1 might be a marker of atherosclerotic lesions in T2D patients with atherosclerosis ${ }^{[43]}$. Salvianolic acid $B$ attenuated the expression of VCAM1 in a dose-dependent manner and significantly inhibited the adhesion of a human monocytic cell line to human aortic endothelial cells ${ }^{[4]}$. Gin- senoside Rb1 could effectively block the TNF-a-induced overexpression of VCAM1 in human endothelial cells ${ }^{[45]}$. Vascular endothelial growth factor (VEGF) is a multifunctional cytokine that promotes angiogenesis and microvascular permeability. VEGF is believed to play a significant role in the development of diabetic complications. VEGFA variants were associated with the development of diabetic retinopathy ${ }^{[46,47]}$. Ginsenoside Rb1 obviously enhanced the expression of VEGF and enhanced myocardial angiogenesis ${ }^{[48]}$. Ginsenoside Rg1 treat- 
ment significantly enhanced angiogenesis and preserved cardiac function, partly by increasing the expression of hypoxiainducible factor 1 (HIF-1) and VEGF ${ }^{[49]}$. Ginsenoside Rg1 also increased the secretion of VEGF by endothelial progenitor cells $^{[50]}$. Crude extracts of Salvia miltiorrhiza and salvianolic acid $B$ enhanced angiogenic processes in mouse endothelial SVR cells through the upregulation of VEGF and VEGF receptor genes ${ }^{[51]}$. However, berberine was reported to mediate antiangiogenic activity through the down-regulation of HIF1 , VEGF, and proinflammatory mediators ${ }^{[52]}$. XKA led to the upregulation of VEGFA. Therefore, salvianolic acid B, ginsenoside $\mathrm{Rg} 1$ and ginsenoside $\mathrm{Rb} 1$ are currently considered to overcome berberine in the regulation of VEGFA.

Some potential targets were affected by more than one compound, while many compounds targeted several genes or proteins. Multiple components of XKA affected numerous targets related to a series of processes involved in progressive disorders; these components would exert synergistic therapeutic effects on T2D.

Many pathways are related to T2D, and pathways related to energy metabolism, insulin resistance, oxidative stress, inflammation, apoptosis processes and vascular disease were all enriched by the related targets. Therefore, these associated mechanisms of action could be the main therapeutic mechanisms of XKA for T2D. The findings regarding XKA in this study provide scientific evidence for the clinical use of XKA for treating T2D. This work also presents the possibility of new indications for XKA in clinical use, such as diabetic vascular complications, which could be further studied.

\section{Conclusion}

In this study, compound-target-pathway networks were constructed to illustrate the therapeutic mechanisms of XKA for T2D and to reveal the active compounds of this Chinese medicine in the treatment of T2D. The therapeutic effects of XKA for T2D may be achieved mainly by improving carbohydrate and lipid metabolism as well as ameliorating insulin resistance, oxidation, and inflammation pathways.

\section{Acknowledgements}

This work was partially financially supported by the National Basic Research Program of China (№ 2012CB518405). The authors sincerely thank the Laboratory Animal Center of Zhejiang University for technical assistance with the animal experiments.

\section{Author contribution}

Zhen-zhong YANG, Zheng LI, and Yi-yu CHENG designed this study, Zhen-zhong YANG and Feng ZHANG conducted the experiments, Zhen-zhong YANG and Wei LIU analyzed the gene expression data, and Zhen-zhong YANG and Zheng LI wrote the manuscript.

\section{Supplementary information}

Supplementary information is available at the Acta Pharmacologica Sinica website.

\section{References}

1 Qi L, Hu FB, Hu G. Genes, environment, and interactions in prevention of type 2 diabetes: a focus on physical activity and lifestyle changes. Curr Mol Med 2008; 8: 519-32.

2 Tahrani AA, Bailey CJ, Del Prato S, Barnett AH. Management of type 2 diabetes: new and future developments in treatment. Lancet 2011; 378: 182-97.

3 Yang Z, Yang J, Liu W, Wu L, Xing L, Wang Y, et al. T2D@ZJU: a knowledgebase integrating heterogeneous connections associated with type 2 diabetes mellitus. Database (Oxford) 2013; 2013: bat052.

4 Timmers S, Hesselink MK, Schrauwen P. Therapeutic potential of resveratrol in obesity and type 2 diabetes: new avenues for health benefits? Ann N Y Acad Sci 2013; 1290: 83-9.

5 Tian P. Convergence: where west meets east. Nature 2011; 480: S84-S6.

6 Nissen SE, Wolski K. Effect of rosiglitazone on the risk of myocardial infarction and death from cardiovascular causes. New Engl J Med 2007; 356: 2457-71.

7 Scarpello J, Hodgson E, Howlett $\mathrm{H}$. Effect of metformin on bile salt circulation and intestinal motility in type 2 diabetes mellitus. Diabet Med 1998; 15: 651-6.

8 Owen M, DORAN E, Halestrap A. Evidence that metformin exerts its anti-diabetic effects through inhibition of complex 1 of the mitochondrial respiratory chain. Biochem J 2000; 348: 607-14.

9 Tian P. CONVERGENCE Where West meets East. Nature 2011; 480: S84-S6.

10 Yang Z, Wang Y, Wang Y, Zhang Y. Bioassay-guided screening and isolation of alpha-glucosidase and tyrosinase inhibitors from leaves of Morus alba. Food Chem 2012; 131: 617-25.

11 Wang Z, Liu J, Cheng Y, Wang Y. Fangjiomics: in search of effective and safe combination therapies. J Clin Pharmacol 2011; 51: 113251.

12 Fang $\mathrm{H}$, Harris SC, Su Z, Chen M, Qian F, Shi L, et al. ArrayTrack: an FDA and public genomic tool. Methods Mol Biol 2009; 563: 379-98.

13 Wang L, Li Z, Zhao X, Liu W, Liu Y, Yang J, et al. A network study of Chinese medicine Xuesaitong injection to elucidate a complex mode of action with multicompound, multitarget, and multipathway. Evid Based Complement Alternat Med 2013; 2013: 652373.

14 Kanehisa M, Goto S, Sato Y, Furumichi M, Tanabe M. KEGG for integration and interpretation of large-scale molecular data sets. Nucleic Acids Res 2012; 40: D109-14.

15 Wang J, Duncan D, Shi Z, Zhang B. WEB-based GEne SeT AnaLysis Toolkit (WebGestalt): update 2013. Nucleic Acids Res 2013; 41: W77-83.

16 Chen X, Zhou H, Liu Y, Wang J, Li H, Ung C, et al. Database of traditional Chinese medicine and its application to studies of mechanism and to prescription validation. Br J Pharmacol 2006; 149: 1092-103.

17 Ye H, Ye L, Kang H, Zhang D, Tao L, Tang K, et al. HIT: linking herbal active ingredients to targets. Nucleic Acids Res 2011; 39: D1055-9.

18 Chen CYC. TCM Database@ Taiwan: the world's largest traditional Chinese medicine database for drug screening in silico. PloS One 2011; 6: e15939.

19 Xue R, Fang Z, Zhang M, Yi Z, Wen C, Shi T. TCMID: traditional Chinese medicine integrative database for herb molecular mechanism analysis. Nucleic Acids Res 2013; 41: D1089-95.

20 Smoot ME, Ono K, Ruscheinski J, Wang PL, Ideker T. Cytoscape 2.8: new features for data integration and network visualization. Bioinformatics 2011; 27: 431-2.

21 Li H, Zhao L, Zhang B, Jiang Y, Wang X, Guo Y, et al. A network pharmacology approach to determine active compounds and action 
mechanisms of Ge-Gen-Qin-Lian decoction for treatment of type 2 diabetes. Evid Based Complement Alternat Med 2014; 2014: 495840.

22 Yu YL, Lu SS, Yu S, Liu YC, Wang P, Xie L, et al. Huang-Lian-Jie-DuDecoction modulates glucagon-like peptide-1 secretion in diabetic rats. J Ethnopharmacol 2009; 124: 444-9.

23 Kong WJ, Zhang H, Song DQ, Xue R, Zhao W, Wei J, et al. Berberine reduces insulin resistance through protein kinase $\mathrm{C}$-dependent upregulation of insulin receptor expression. Metabolism 2009; 58 : 109-19.

24 Zhang H, Wei J, Xue R, Wu JD, Zhao W, Wang ZZ, et al. Berberine lowers blood glucose in type 2 diabetes mellitus patients through increasing insulin receptor expression. Metabolism 2010; 59: 28592.

25 Kim SH, Shin EJ, Kim ED, Bayaraa T, Frost SC, Hyun CK. Berberine activates GLUT1-mediated glucose uptake in 3T3-L1 adipocytes. Biol Pharm Bull 2007; 30: 2120-5.

26 Cok A, Plaisier C, Salie MJ, Oram DS, Chenge J, Louters LL. Berberine acutely activates the glucose transport activity of GLUT1. Biochimie 2011; 93: 1187-92.

27 Xing J, Kang L, Jiang Y. Effect of dietary betaine supplementation on lipogenesis gene expression and CpG methylation of lipoprotein lipase gene in broilers. Mol Biol Reports 2011; 38: 1975-81.

28 Guo HW, Yun CX, Hou GH, Du J, Huang X, Lu Y, et al. Mangiferin attenuates Th1/Th2 cytokine imbalance in an ovalbumin-induced asthmatic mouse model. PloS One 2014; 9: e100394.

29 Fu K, Piao T, Wang M, Zhang J, Jiang J, Wang X, et al. Protective effect of catalpol on lipopolysaccharide-induced acute lung injury in mice. Int Immunopharmacol 2014; 23: 400-6.

30 Liu Y, Qi W, Richardson A, Van Remmen H, Ikeno Y, Salmon AB. Oxidative damage associated with obesity is prevented by overexpression of CuZn-or Mn-superoxide dismutase. Biochem Biophys Res Commun 2013; 438: 78-83.

31 Tinahones FJ, MurriPierri M, Garrido-Sánchez L, García-Almeida JM, García-Serrano S, García-Arnés J, et al. Oxidative stress in severely obese persons is greater in those with insulin resistance. Obesity 2009; 17: 240-6.

32 Muscogiuri G, Salmon AB, Aguayo-Mazzucato C, Li M, Balas B, Guardado-Mendoza R, et al. Genetic disruption of SOD1 gene causes glucose intolerance and impairs $\beta$-cell function. Diabetes 2013; 62 : 4201-7.

33 Li Y, Bao Y, Jiang B, Wang Z, Liu Y, Zhang C, et al. Catalpol protects primary cultured astrocytes from in vitro ischemia-induced damage. Int J Develop Neurosci 2008; 26: 309-17.

34 Tang Y, Wang M, Le X, Meng J, Huang L, Yu P, et al. Antioxidant and cardioprotective effects of Danshensu (3-(3,4-dihydroxyphenyl)-2hydroxy-propanoic acid from Salvia miltiorrhiza) on isoproterenolinduced myocardial hypertrophy in rats. Phytomedicine 2011; 18 : 1024-30.

35 Sellamuthu PS, Arulselvan P, Kamalraj S, Fakurazi S, Kandasamy M. Protective nature of mangiferin on oxidative stress and antioxidant status in tissues of streptozotocin-induced diabetic rats. ISRN Pharmacol 2013; 2013.

36 Zhang YX, Wang L, Xiao EL, Li SJ, Chen JJ, Gao B, et al. GinsenosideRd exhibits anti-inflammatory activities through elevation of antioxidant enzyme activities and inhibition of JNK and ERK activation in vivo. Int Immunopharmacol 2013; 17: 1094-100.
37 Bitar MS, Al-Mulla F. A defect in Nrf2 signaling constitutes a mechanism for cellular stress hypersensitivity in a genetic rat model of type 2 diabetes. Am J Physiol-Endocrinol Metab 2011; 301: E1119-E29.

38 Chartoumpekis DV, Kensler TW. New player on an old field; the Keap1/Nrf2 pathway as a target for treatment of type 2 diabetes and metabolic syndrome. Curr Diabetes Rev 2013; 9: 137.

39 Chong CM, Zhou ZY, Razmovski-Naumovski V, Cui GZ, Zhang LQ, Sa $\mathrm{F}$, et al. Danshensu protects against 6-hydroxydopamine-induced damage of PC12 cells in vitro and dopaminergic neurons in zebrafish. Neuros Lett 2013; 543: 121-5.

40 Zhang H, Liu YY, Jiang Q, Li KR, Zhao YX, Cao C, et al. Salvianolic acid A protects RPE cells against oxidative stress through activation of Nrf2/HO-1 signaling. Free Radical Biol Med 2014; 69: 219-28.

41 Zhou J, Qu XD, Li ZY, Liu Q, Ma YH, He JJ. Salvianolic acid B attenuates toxin-Induced neuronal damage via Nrf2-dependent glial cells-mediated protective activity in Parkinson's disease models. PloS One 2014; 9: e101668.

42 Kado S, Nagata N. Circulating intercellular adhesion molecule-1, vascular cell adhesion molecule-1, and E-selectin in patients with type 2 diabetes mellitus. Diabetes Res Clin Pract 1999; 46: 143-8.

43 Otsuki M, Hashimoto K, Morimoto Y, Kishimoto T, Kasayama S. Circulating vascular cell adhesion molecule-1 (VCAM-1) in atherosclerotic NIDDM patients. Diabetes 1997; 46: 2096-101.

44 Chen YH, Lin SJ, Ku HH, Shiao MS, Lin FY, Chen JW, et al. Salvianolic acid $B$ attenuates VCAM-1 and ICAM-1 expression in TNF-alphatreated human aortic endothelial cells. J Cell Biochem 2001; 82: 512-21.

45 Chai H, Wang Q, Huang L, Xie T, Fu Y. Ginsenoside Rb1 inhibits tumor necrosis factor-alpha-induced vascular cell adhesion molecule-1 expression in human endothelial cells. Biol Pharm Bull 2008; 31: 2050-6.

46 Al-Kateb H, Mirea L, Xie X, Sun L, Liu M, Chen H, et al. Multiple variants in vascular endothelial growth factor (VEGFA) are risk factors for time to severe retinopathy in type 1 diabetes the DCCT/EDIC genetics study. Diabetes 2007; 56: 2161-8.

47 Abhary S, Burdon KP, Gupta A, Lake S, Selva D, Petrovsky N, et al. Common sequence variation in the VEGFA gene predicts risk of diabetic retinopathy. Invest Ophthalmol Visual Sci 2009; 50: 5552-8.

48 Liu Y, Liu S, Liu Z. Eeffects of ginsenoside Rbl on blood vessel regeneration after ischemia and reperfusion in rats. Chin $\mathrm{J}$ Histochem Cytochem 2008; 17: 39-44.

49 Zhang YJ, Zhang XL, Li MH, Iqbal J, Bourantas CV, Li JJ, et al. The ginsenoside Rg1 prevents transverse aortic constriction-induced left ventricular hypertrophy and cardiac dysfunction by inhibiting fibrosis and enhancing angiogenesis. J Cardiovasc Pharmacol 2013; 62: 50-7.

50 Shi AW, Wang XB, Lu FX, Zhu MM, Kong XQ, Cao KJ. Ginsenoside Rg1 promotes endothelial progenitor cell migration and proliferation. Acta Pharmacol Sin 2009; 30: 299-306.

51 Lay IS, Chiu JH, Shiao MS, Lu WY, Wu CW. Crude extract of Salvia miltiorrhiza and salvianolic acid $\mathrm{B}$ enhance in vitro angiogenesis in murine SVR endothelial cell line. Planta Med 2003; 69: 26-32.

52 Hamsa TP, Kuttan G. Antiangiogenic activity of berberine is mediated through the downregulation of hypoxia-inducible factor-1, VEGF, and proinflammatory mediators. Drug Chem Toxicol 2012; 35: 57-70. 